\title{
The Proprioceptive Neuromuscular Facilitation Reduces Isometric Strength in Apparently Health Women
}

\author{
Gabriel Costa e Silva ${ }^{1,2 *}$, Rodrigo Rodrigues ${ }^{3}$, Anderson Silveira ${ }^{4}$, Fabrízio Di Masi ${ }^{4}$, Carlos Herdy ${ }^{5}$ and Roberto \\ Simão $^{6}$
}

${ }^{1}$ Faculdade de Medicina do ABC, Fundação ABC, Brazil

${ }^{2}$ Grupo de Pesquisa em Ciências do Movimento Humano, Colégio Pedro II, Brazil

${ }^{3}$ Departamento de Medicina, Universidade Federal de São Paulo, Brazil

${ }^{4}$ Departamento de Educação Física e Desporto, Universidade Federal Rural do Rio de Janeiro, Brazil

${ }^{5}$ Programa de Pós-Graduação em Epidemiologia e Saúde Pública, Fundação Oswaldo Cruz, Brazil

${ }^{6}$ Escola de Educação Física e Desportos, Universidade Federal do Rio de Janeiro, Brazil

*Corresponding author: Gabriel Costa e Silva, Programa de Doutorado em Ciências da Saúde, Faculdade de Medicina do ABC, Fundação ABC, Brazil

Submission: 眥 April 16, 2018; Published: 阱 May 29, 2018

\section{Short Communication}

Muscular strength is considered one of the principal physical aptitude and healthy components [1] and its evaluation through manual grip, besides being relatively simple, does not involve considerable financial expenditures and possesses good predictive capacity. Therefore, it can have great value for triage in programs and researches considering physical activities and sports [2]. Suitable levels of strength and flexibility are important for good physical aptitude and healthy1 thereby previous works report stretching capacity in sport performance reduction [3], by neural and structural modifications due to muscular stretching [4]. Thus, this work aims to investigate the acute effect of PNF stretching method on the isometric strength of muscles used in manual grip of healthy women.

Twenty seven ( $\mathrm{n}=27)$ subjects started the study. However, after going through inclusion and exclusion criterias, thirteen $(n=13)$ women apparently healthy (with menstrual cycle controlled) were used as sample of the present study. This research project was submitted and approved by Comitê de Ética na Pesquisa da Universidade Federal Rural do Rio de Janeiro (protocol number: 23083.006771/2011-02).

$1^{\text {st }}$ Visit: Individuals signed the written consent forms, underwent an anthropometric evaluation for the respective measures: body mass, stature, body composition (percentage of body fat) and evaluation of flexibility levels through goniometric evaluation (Lafayette Goniometer, EUA). Stature was measured with standing participants, barefoot, using a stadiometer (Sanny, $0.1 \mathrm{~cm}$, Brasil). Body mass was measured by a digital scale (Plenna, $0.1 \mathrm{~kg}$, USA). Body composition and flexibility were also evaluated and lastly, familiarization to the isometric strength test was done using a hand gripp (Jaymar 5030JI, USA).

$2^{\text {nd }}$ Visit-Using a hand gripp, three consecutive attempts were done, with a 60 seconds rest in between, the higher value was being considered as 1RM [5].

$3^{\text {rd }}$ Visit-Sample was randomly divided in 2 situations: 1) with pre-exercise stretching (PNF) and other with no stretching (NS). PNF subjects performed 1RM manual grip maximum test preceded by stretching wrist flexor muscles through PNF method, according to Costa e Silva et al. [6].

$4^{\text {th }}$ Visit - Respecting 48 hours between visits, protocols were randomly repeated until all subjects had their tests done in the 2 whole proposed situations. Always before tests, all individuals had their body temperature measured by forehead digital thermometers (Microlife MIT-FR1DMI, USA) and had shown an average of $35.85 \pm 1.07^{\circ} \mathrm{C}$. Visits were conducted in laboratory and, previously to tests, average values of air humidity were written down (55-60\%) and room temperature $\left(20-23^{\circ} \mathrm{C}\right)$.

In order to determine treatment effects on a dependent variable (maximum strength of manual grip maximum) test $t$ Student significance level adopted was $\mathrm{p} \leq 0.05$. Calculation on isometric strength changes after PNF has been realized through effect impact 
(effect size) (difference between experimental average and control, divided by control standard deviation, proposed by Rhea [7].

Results found in the present study have shown statistically significant differences between PNF and NS $(21.53 \pm 6.38$ vs $23.53 \pm 6.06 \mathrm{Kg} / \mathrm{N}$; $\mathrm{p}=0.039$ ) (Figure 1). Between situations NS vs. PNF, the impact effect (0.33) proved trivial magnitude of isometric strength alteration (Table 1). Our study has demonstrated that stretching through PNF reduces significantly the capacity to produce isometric strength in muscles related to manual grip.
There are hypothesis described in the literature suggesting that the usage of muscular stretching before exercising causes reduction on performance in several tests, mainly related to immediate strength production4, this could be related to factors as length-tension, neural and mechanical aspects being responsible for possible losses of muscular strength performance [8]. Besides this, a study of our group demonstrated that stretching, done by static method, was able to reduce the capacity of producing isometric strength in 25 subjects with experience on strength training Costa e Silva et al. [6].

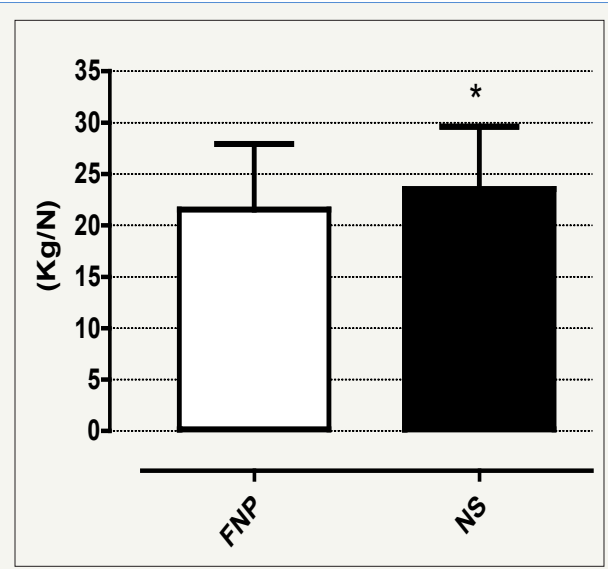

Figure 1: Mean and standard deviation of isometric strength $(\mathrm{Kg} / \mathrm{N})$ in $1 \mathrm{RM}$ manual grip maximum test, preceded or not by PNF (PNF vs. NS) NS: No Stretching Situation; PNF: Proprioceptive Neuromuscular Facilitation. ${ }^{*}(\mathrm{p}<0.05)$.

Table 1: Effect size and magnitude of change in the static muscle strength between situations.

\begin{tabular}{|c|c|}
\hline & NS vs PNF \\
\hline Effect Size & 0.33 \\
\hline Magnitude & Trivial \\
\hline
\end{tabular}

NS: No Stretching Situation; PNF: Proprioceptive Neuromuscular Facilitation

According to our results, 30 seconds of stretching by PNF method is already enough to occasion sensibility decrease in muscular proprioceptors, tendinous and articular, as well as in nociceptors, that constitute fundamental mechanisms for protection and organization of structures related to human movement, such strength reduction after stretching in different insistence times can be, partially, explained by an autogenic inhibition and reciprocal inhibition caused by stretching due to Golgi tendon organs activation that can cause decrease in alpha motoneurons excitability. However, corroboring with Chagas et al. [9], we recommend further studies involving the long-term effects of stretching using different stretching methods and neurophysiological variables. In summary, it was verified that acutely stretching method by PNF causes reduction on muscular isometric strength of muscles involved in manual grip.

\section{References}

1. Garber CE, Blissmer B, Deschenes MR, Franklin BA, Lamonte MJ, et al (2011) Quantity and quality of exercise for developing and maintain cardiorespiratory, musculoskeletal, and neuromotor fitness in apparently healthy adults: guidance for prescribing exercise. Med Sci Sport Exerc 43: 1334-1359.

2. Visnapuu M, Jurimae T (2007) Handgrip strength and hand dimensions in young handball and basketball players. J Strength Cond Res 21(3): 923-929.

3. Nelson AG, Driscoll NM, Landin DK, Young MA, Schexnayder IC (2005) Acute effects of passive muscle stretching on sprint performance. J Strength Cond Res 23(5): 449-454.

4. Rubini EC, Costa ALL, Gomes PSC (2007) The effects of stretching on strength performance. Sports Med 37(3): 213-224.

5. Sale DG, Jacobs I, MacDougall JD, Garner S (1990) Comparison of two regimens of concorrent strength and endurance training. Med Sci Sports Exerc 22(3): 348-356.

6. Costa e Silva G, Silveira ALB, Di Masi, F, Bentes CM, Miranda HL, et al. (2012) Acute effect of static stretching on isometric muscle strength performance. ConScientiae Saúde 11: 274-280.

7. Rhea MR (2004) Determining the magnitude of treatment effects in strength training research through the use of the effect size. J Strength Cond Res 18(4): 918-920.

8. Shirier I (2004) Does stretching improve performance? A systematic and critical review of the literature. Clin J Sports Med 14(5): 267-273.

9. Chagas MH, Magalhães FA, Peixoto GH, Pereira BM, Andrade AG, et al. (2016) Exploratory factor analysis for differentiating sensory and mechanical variables related to muscle-tendon unit elongation. Braz J Phys Ther 20: 240-247. 
Creative Commons Attribution 4.0 International License

For possible submissions Click Here
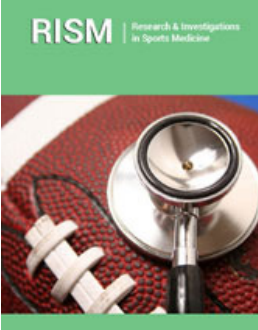

Research \& Investigations in Sports Medicine

\section{Benefits of Publishing with us}

- High-level peer review and editorial services

- Freely accessible online immediately upon publication

- Authors retain the copyright to their work

- Licensing it under a Creative Commons license

- Visibility through different online platforms 